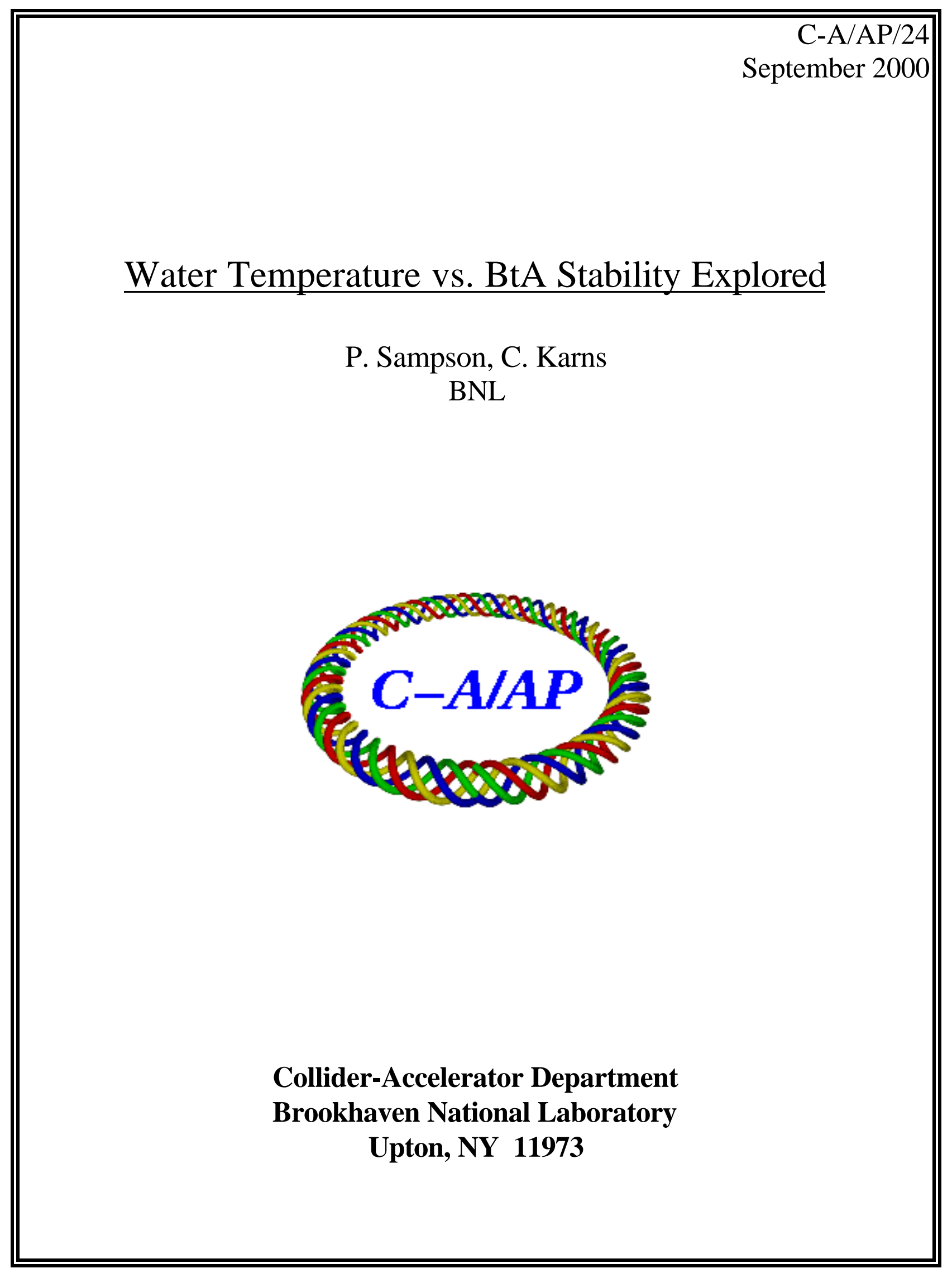




\begin{tabular}{|l||}
\hline \multicolumn{1}{|c||}{ AGS Complex Machine Studies } \\
Water Temperature VS. BtA stability Explored \\
\hline Study Period: February $18^{\text {th }}, 2000$ \\
\hline Participants: C. Karns \\
Reported by: P. Sampson, C. Karns \\
\hline Machine Booster and BtA \\
\hline Beam: High Intensity Protons \\
\hline Tools: CBMs, Spreadsheet, BtA Harps and Booster Gauss Clock \\
\hline $\begin{array}{l}\text { Aim: To study the effect that the water temperature change brought on by turning the Booster Main } \\
\text { Magnet off has on BtA and transfer efficiency. }\end{array}$ \\
\hline
\end{tabular}

\section{Water Temperature vs. BTA stability explored}

\section{Summary}

This experiment was performed as part of the ongoing effort to determine the source of unexpected steering changes in the Booster to AGS (BtA) line. Since it was commissioned, this line has proven to need steering adjustments at regular intervals. Also of concern is that beam steering does not return to nominal when a file is loaded following any changes to the line (i.e. after a study). One thought was that changes in cooling water temperature, brought on by turning the Booster Main Magnet power supply (BMMPS) off, effect BtA transport elements, since many of these devices are cooled by the same system.

It was observed that turning off the BMMPS, made its cooling water temperature drop from $98^{\circ} \mathrm{f}$ to $92^{\circ} \mathrm{f}$ or $6 \%$. There was no observable effect on power supply read backs for elements in the BtA line, however, changes were seen in the Booster Main Ring injection field. Further study is indicated.

\section{Experiment}

Transport between the AGS and Booster was optimized, then archived. Data for power supply read backs, Booster Main Ring fields, BtA harp positions and Booster extraction radius were recorded. The BMMPS was then turned off and the water temperature allowed to stabilize. Once stable, the MMPS was re-energized and sets of data for the above parameters were taken every 15 minutes until 
the cooling water reached normal operating temperature. A total of 5 sets of data were taken. Intensities were recorded throughout the exercise.

\section{Results}

SpreadSheet read backs vs. Time for BtA elements:

\begin{tabular}{|c|c|c|c|c|c|c|c|c|c|}
\hline Time & DH1 & DH2-3 & DH4 & DH127 & DH158 & DH5 & DV007 & DV030 & DV120 \\
\hline $\mathbf{1 0 : 0 0}$ & 183.08 & 4212.5 & 172.601 & 14.7582 & 4.0256 & 526.99 & 0.9267 & -1.5275 & 0.5604 \\
\hline $\mathbf{1 1 : 0 0}$ & 183.76 & 4208.8 & 172.381 & 14.7948 & 4.0256 & 527.97 & 0.9194 & -1.5861 & 0.5604 \\
\hline $\mathbf{1 1 : 1 5}$ & 182.56 & 4212.5 & 172.308 & 14.7729 & 4.0183 & 527.24 & 0.9267 & -1.5495 & 0.5604 \\
\hline $\mathbf{1 1 : 3 0}$ & 182.22 & 4212.5 & 171.868 & 14.7729 & 4.0183 & 526.75 & 0.9267 & -1.5568 & 0.5677 \\
\hline $\mathbf{1 1 : 4 5}$ & 183.59 & 4212.5 & 172.527 & 14.7655 & 4.011 & 527.24 & 0.934 & -1.5568 & 0.5677 \\
\hline $\mathbf{1 2 : 0 0}$ & 183.42 & 4212.5 & 172.454 & 14.7655 & 4.0256 & 527.24 & 0.9121 & -1.5128 & 0.5531 \\
\hline
\end{tabular}

\begin{tabular}{|c|c|c|c|c|c|c|c|c|c|}
\hline Time & DV181 & QV1 & QH2 & QV3 & QH4 & QV5 & QH6 & QV7 & QH8 \\
\hline $\mathbf{1 0 : 0 0}$ & 7.8644 & 427.6 & 533.1 & 511.12 & 675.95 & 608.43 & 641.4 & 323.82 & 324.18 \\
\hline $\mathbf{1 1 : 0 0}$ & 7.8425 & 427.85 & 532.85 & 511.36 & 680.1 & 607.7 & 641.4 & 323.45 & 329.18 \\
\hline $\mathbf{1 1 : 1 5}$ & 7.8644 & 427.6 & 532.85 & 510.87 & 675.46 & 608.07 & 641.03 & 322.72 & 324.72 \\
\hline $\mathbf{1 1 : 3 0}$ & 7.8718 & 427.36 & 533.1 & 510.87 & 675.71 & 608.43 & 640.67 & 323.45 & 324.3 \\
\hline $\mathbf{1 1 : 4 5}$ & 7.8718 & 427.36 & 532.85 & 510.63 & 674.97 & 607.33 & 641.4 & 323.45 & 324.05 \\
\hline $\mathbf{1 2 : 0 0}$ & 7.8571 & 427.6 & 532.85 & 511.12 & 678.39 & 607.7 & 641.4 & 323.82 & 323.93 \\
\hline
\end{tabular}

\begin{tabular}{|c|c|c|c|c|c|c|c|}
\hline Time & QV9 & QH10 & QV11 & QH12 & QV13 & QH14 & QH15 \\
\hline $\mathbf{1 0 : 0 0}$ & 519.66 & 147.35 & 258.242 & 378.97 & 560.45 & 318.69 & 0.49 \\
\hline $\mathbf{1 1 : 0 0}$ & 517.09 & 144.96 & 258.242 & 376.75 & 558.25 & 330.17 & 1.59 \\
\hline $\mathbf{1 1 : 1 5}$ & 520.17 & 143.59 & 258.315 & 377.26 & 559.23 & 325.29 & 1.34 \\
\hline $\mathbf{1 1 : 3 0}$ & 518.8 & 146.84 & 258.168 & 377.61 & 560.93 & 317.47 & 1.34 \\
\hline $\mathbf{1 1 : 4 5}$ & 518.63 & 147.01 & 258.022 & 378.63 & 560.69 & 318.2 & 0.98 \\
\hline $\mathbf{1 2 : 0 0}$ & 519.14 & 146.84 & 258.242 & 379.14 & 559.96 & 319.67 & 2.44 \\
\hline
\end{tabular}



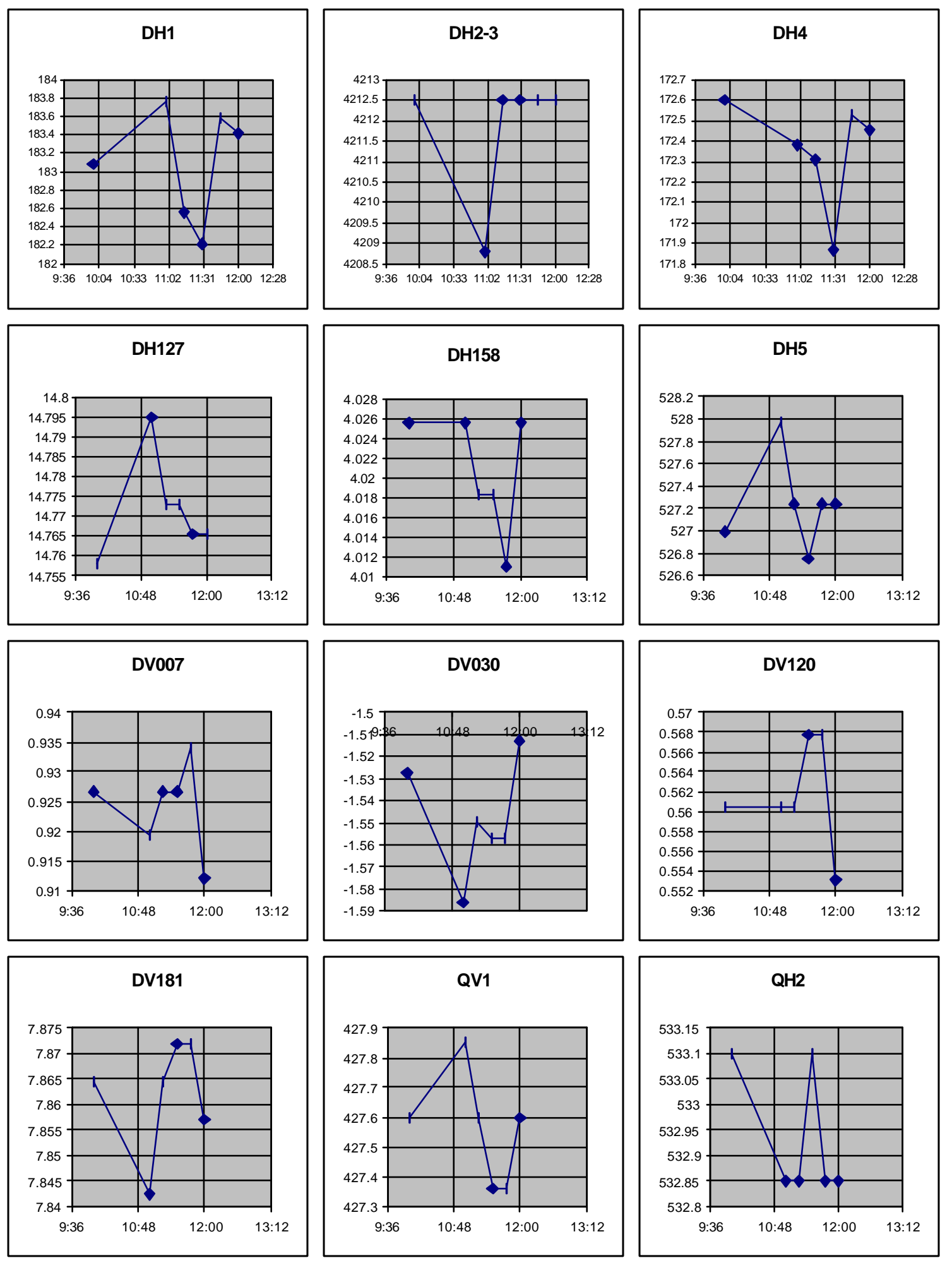

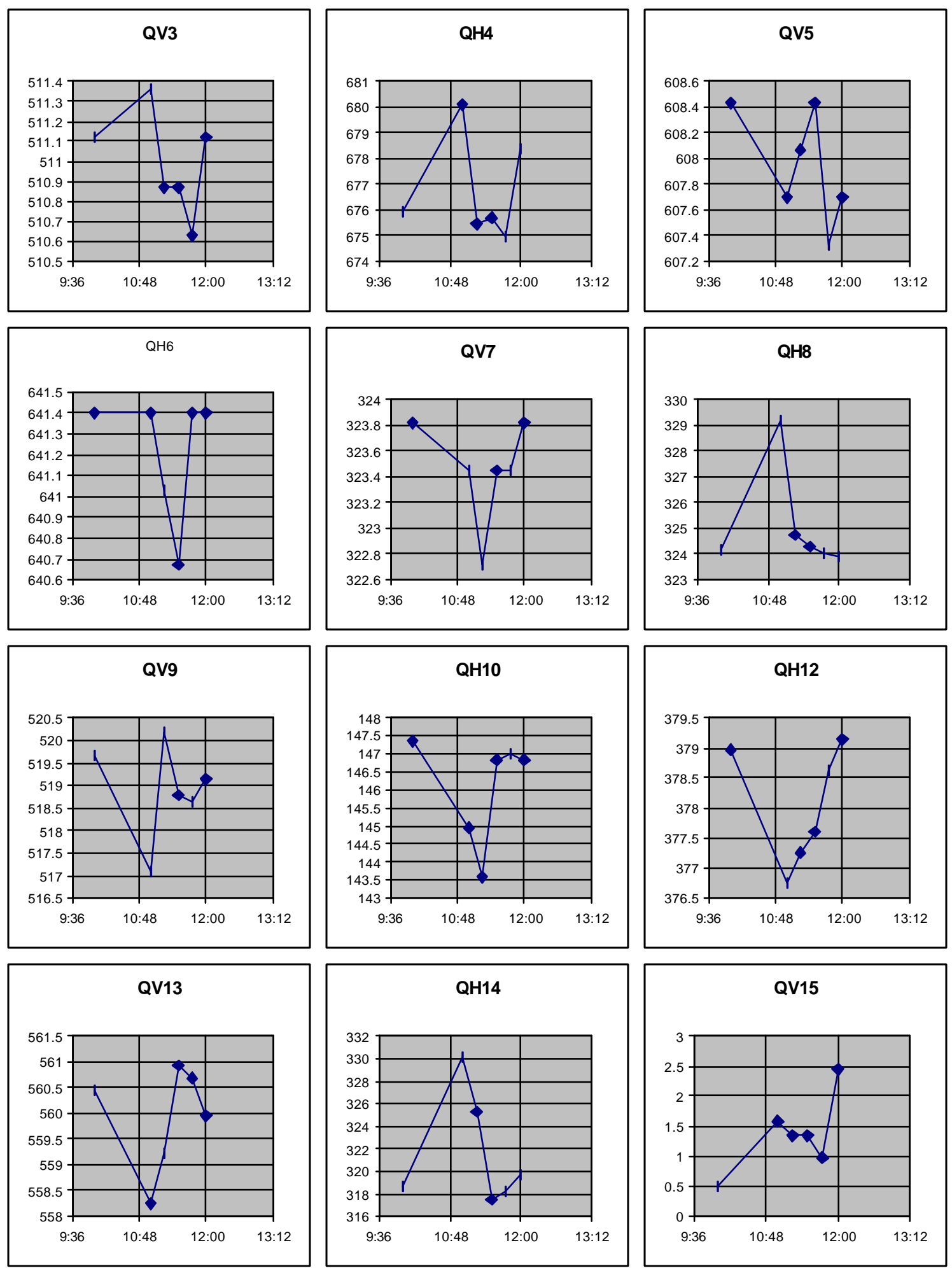
Position of beam envelope in BtA vs. Time (no changes in width were observed):

\begin{tabular}{|r|r|r|r|r|r|r|}
\hline MW006 & & & & & MW 060 & \\
\hline & H mean & V mean & & & H mean & V mean \\
\hline $10: 00$ & 1.36 & 1.09 & & $10: 00$ & -19.31 & 0.72 \\
\hline $11: 00$ & 2.17 & 0.98 & & $11: 00$ & -16.58 & 0.72 \\
\hline $11: 15$ & 1.98 & 1.03 & & $11: 15$ & -16.24 & 0.7 \\
\hline $11: 30$ & 1.9 & 1.02 & & $11: 30$ & -16.63 & 0.72 \\
\hline $11: 45$ & 2.01 & 1.01 & & $11: 45$ & -15.95 & 0.74 \\
\hline $12: 00$ & 1.98 & 1.02 & & $12: 00$ & -15.74 & 0.71 \\
\hline MW 125 & & & & MW 166 & & \\
\hline & $H$ mean & V mean & & & H mean & V mean \\
\hline $10: 00$ & -6.9 & 3.12 & & $10: 00$ & -6.79 & -4.84 \\
\hline $11: 00$ & -5.03 & 3.2 & & $11: 00$ & -6.51 & -4.39 \\
\hline $11: 15$ & -5 & 2.98 & & $11: 15$ & -6.52 & -4.75 \\
\hline $11: 30$ & -5.44 & 3.59 & & $11: 30$ & -5.94 & -4.49 \\
\hline $11: 45$ & -4.67 & 2.93 & & $11: 45$ & -6.21 & -4.36 \\
\hline $12: 00$ & -5.33 & 2.29 & & $12: 00$ & -6.32 & -4.88 \\
\hline
\end{tabular}
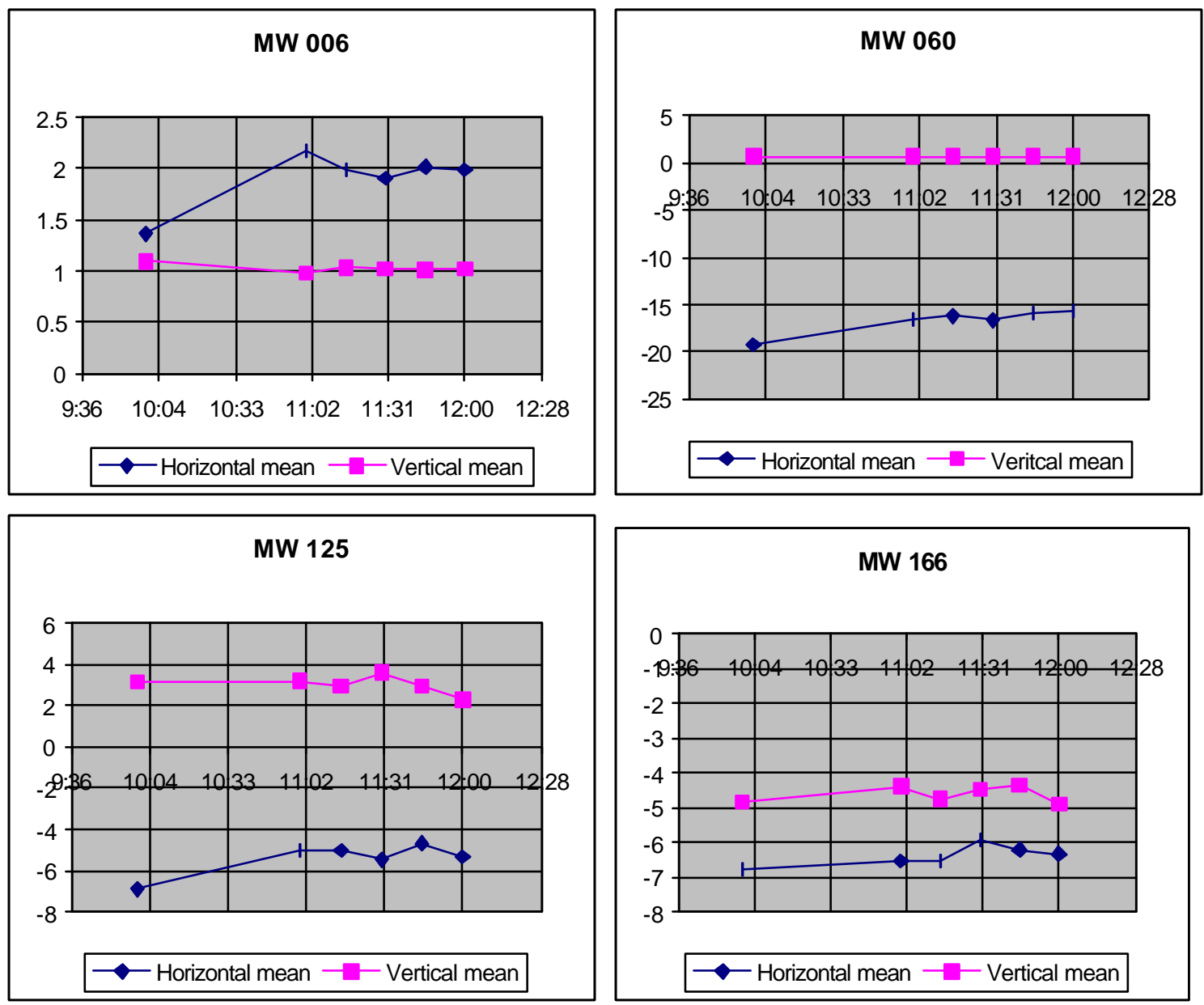
The following section shows how the overall efficiency behaved during the study period, each instance is representative of scalar reading on a single pulse:

Scalar read backs vs. Time:

\begin{tabular}{|r|r|r|r|r|r|}
\hline Time & \multicolumn{1}{|c|}{ BSTR input } & \multicolumn{1}{c|}{ BSTR early } & \multicolumn{1}{c|}{ BSTR late } & \multicolumn{1}{c|}{ AGS CBM } & BTA efficiency \\
\hline $10: 00$ & $1.72 \mathrm{E}+13$ & $1.34 \mathrm{E}+13$ & $1.24 \mathrm{E}+13$ & $1.06 \mathrm{E}+13$ & $85.48 \%$ \\
\hline $11: 00$ & $1.70 \mathrm{E}+13$ & $1.26 \mathrm{E}+13$ & $1.12 \mathrm{E}+13$ & $6.88 \mathrm{E}+12$ & $61.43 \%$ \\
\hline $11: 15$ & $1.71 \mathrm{E}+13$ & $1.27 \mathrm{E}+13$ & $1.11 \mathrm{E}+13$ & $9.42 \mathrm{E}+12$ & $84.86 \%$ \\
\hline $11: 30$ & $1.72 \mathrm{E}+13$ & $1.26 \mathrm{E}+13$ & $1.11 \mathrm{E}+13$ & $9.28 \mathrm{E}+12$ & $83.60 \%$ \\
\hline $11: 45$ & $1.71 \mathrm{E}+13$ & $1.27 \mathrm{E}+13$ & $1.12 \mathrm{E}+13$ & $9.50 \mathrm{E}+12$ & $84.82 \%$ \\
\hline $12: 00$ & $1.71 \mathrm{E}+13$ & $1.27 \mathrm{E}+13$ & $1.12 \mathrm{E}+13$ & $9.36 \mathrm{E}+12$ & $83.57 \%$ \\
\hline
\end{tabular}

\section{Transfer efficiency plots}

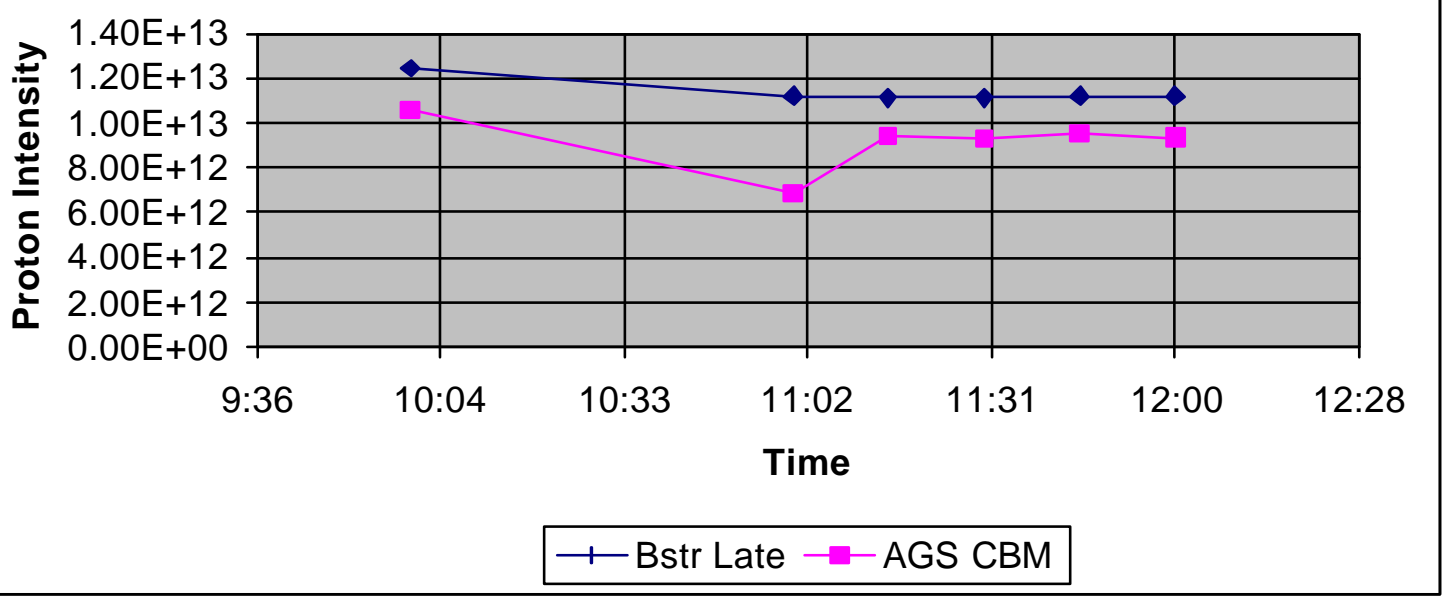


The Booster injection field was also recorded at the Booster's Injection Peaker value several times during the study period.

Gauss clock readings vs. Time

\begin{tabular}{|r|r|}
\hline \multicolumn{2}{|c|}{ Gauss counts } \\
\hline \hline $10: 00$ & 1783 \\
\hline $11: 00$ & 1797 \\
\hline $11: 15$ & 1796 \\
\hline $11: 30$ & 1795 \\
\hline $11: 45$ & 1795 \\
\hline $12: 00$ & 1794 \\
\hline
\end{tabular}

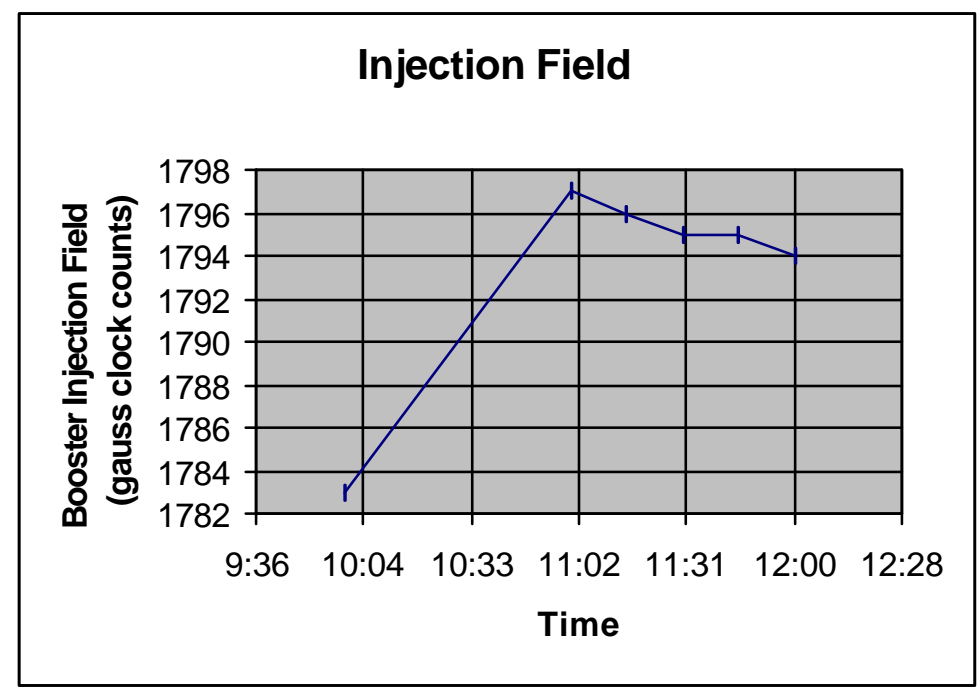

\section{Conclusion:}

Though turning off the Booster Main Magnet power supply does change the cooling water temperature, which is shared by BTA elements, there is no demonstrated proof that this affects the elements in the line. The data shows that before and after the water temperature change, the beam positions and power supply read backs are within normal tolerances. The fact that there appears to be some shift in the Booster's injection field after the supply is turned back on, suggests that further study of this may provide information helpful in solving the BTA instability problem. 